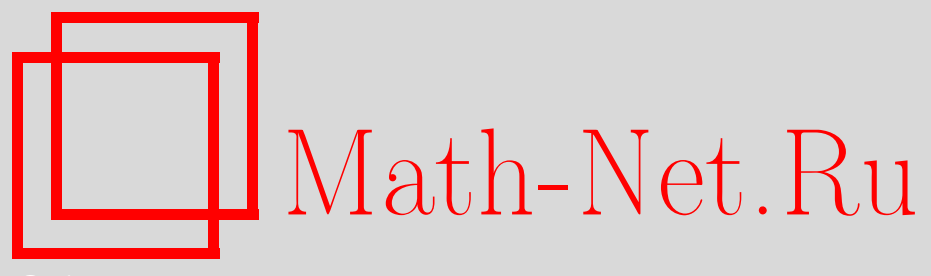

Ли Гу-Цян, Квантовая энтропия полей Дирака в черных дырах, ТМФ, 2006, том 149, номер 1, 60-64

DOI: https://doi.org/10.4213/tmf3830

Использование Общероссийского математического портала Math-Net.Ru подразумевает, что вы прочитали и согласны с пользовательским соглашением http://www . mathnet.ru/rus/agreement

Параметры загрузки:

IP: 54.164 .48 .24

26 апреля 2023 г., 13:17:33

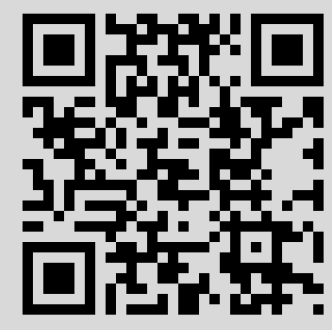




\title{
КВАНТОВАЯ ЭНТРОПИЯ ПОЛЕЙ ДИРАКА В ЧЕРНЫХ ДЫРАХ
}

\begin{abstract}
С использованием модели жесткой стенки исследуется квантовая энтропия дираковских полей в статической черной дыре, содержащей монополь или космическую струну. Показано, что выражение для энтропии поля Дирака содержит квадратично-расходящийся член и два логарифмически-расходящихся члена, поэтому она не пропорциональна энтропии скалярного поля. Вклад логарифмического члена в энтропию зависит от характеристик черной дыры и всегда отрицателен.
\end{abstract}

Ключевые слова: квантовая энтропия, поле Дирака, модель жесткой стенки, черная дыра.

\section{1. ВВЕДЕНИЕ}

Начиная с того момента, как Бекенштейн и Хокинг [1] открыли с помощью сравнения физики черных дыр и термодинамики пропорциональность энтропии черной дыры площади горизонта событий, и с момента открытия эффекта испарения черных дыр были приложены немалые усилия для исследования статистического происхождения энтропии черных дыр [2]-[4]. При этом широко применялся метод “жесткой стенки”, предложенный “т Хоофтом, с использованием которого он впервые исследовал вклад в энтропию черной дыры Шварцшильда, обусловленный присутствием скалярного поля [2]. Впоследствии при помощи этого метода были исследованы скалярные поля в различных фоновых полях черной дыры [3]. Недавно этот же метод был успешно применен при исследованиях структуры расходимостей статистической энтропии статических сферически-симметричных и аксиальносимметричных черных дыр, обусловленных присутствием спиновых полей [4]. Для статической черной дыры с глобальным монополем или с космической струной Джинг [5] исследовал энтропию скалярного поля с использованием модели жесткой стенки и евклидова интеграла по путям, а Лу [6] - энтропию поля Дирака с использованием модели жесткой стенки, но ни один из исследователей не рассматривал логарифмические поправки от следующего порядка пертурбативного разложения.

*School of Information Technology and Science, Zhanjiang Normal College, Zhanjiang, Guangdong, China. E-mail: zsgqli@hotmail.com 
Цель данной работы состоит в изучении квантовой энтропии поля Дирака в статической черной дыре с глобальным полем монополя или с космической струной и исследовании с применением модели жесткой стенки того, как наличие спина у дираковской частицы влияет на эту энтропию.

Метрики снаружи черной дыры с глобальным монополем или с космической струной соответственно имеют вид [5], [6]

$$
\begin{aligned}
& d s^{2}=\left(1-\frac{2 M}{r}\right) d t^{2}-\left(1-\frac{2 M}{r}\right)^{-1} d r^{2}-r^{2}\left(1-8 \pi \eta^{2}\right)\left(d \theta^{2}+\sin ^{2} \theta d \varphi^{2}\right), \\
& d s^{2}=\left(1-\frac{2 M}{r}\right) d t^{2}-\left(1-\frac{2 M}{r}\right)^{-1} d r^{2}-r^{2}\left[d \theta^{2}+\left(1-\mu^{2}\right) \sin ^{2} \theta d \varphi^{2}\right],
\end{aligned}
$$

где $M$ - массовый параметр Шварцшильда, $\eta$ - масштаб нарушения симметрии, а $\mu$ - удельная масса на единицу длины струны.

\section{2. УРАВНЕНИЯ ДЛЯ ПОЛЯ ДИРАКА}

В искривленном пространстве-времени уравнения для безмассового спинорного поля Дирака имеют вид

$$
\begin{gathered}
(D+\varepsilon-\rho) F_{1}+(\bar{\delta}+\pi-\alpha) F_{2}=0, \\
(\Delta+\mu-\gamma) F_{2}+(\delta+\beta-\tau) F_{1}=0, \\
\left(D+\varepsilon^{*}-\rho^{*}\right) G_{2}+\left(\delta+\pi^{*}-\alpha^{*}\right) G_{1}=0, \\
\left(\Delta+\mu^{*}-\gamma^{*}\right) G_{1}+\left(\bar{\delta}+\beta^{*}-\tau^{*}\right) G_{2}=0,
\end{gathered}
$$

где $\Phi=\left(F_{1}, F_{2}, G_{1}, G_{2}\right)^{\mathrm{T}}$ - четырехкомпонентный спинор, $D=l^{\mu} \partial_{\mu}, \Delta=n^{\mu} \partial_{\mu}$, $\delta=m^{\mu} \partial_{\mu}$ - обыкновенные дифференциальные операторы, а $\alpha, \beta, \gamma, \ldots$ - спиновые коэффициенты Ньюмана-Пенроуза.

Рассмотрим статическую сферически-симметричную черную дыру общего вида, метрика которой может быть записана как

$$
d s^{2}=B d t^{2}-B^{-1} d r^{2}-C\left(d \theta^{2}+b \sin ^{2} \theta d \varphi^{2}\right),
$$

где $B=1-2 M / r$ и $b-$ константа.

Чтобы записать уравнения (3) в вышеприведенном пространстве-времени черной дыры, выберем нулевую тетраду в виде

$$
\begin{aligned}
l^{\mu} & =\frac{1}{\sqrt{2 B}}(1,-B, 0,0), \\
n^{\mu} & =\frac{1}{\sqrt{2 B}}(1, B, 0,0), \\
m^{\mu} & =\frac{1}{\sqrt{2 C}}\left(0,0,-1,-i \frac{\operatorname{cosec} \theta}{\sqrt{b}}\right) .
\end{aligned}
$$

Из уравнений (4) и (5) можно получить ненулевые спиновые коэффициенты, если применить формулу Ньюмана-Пенроуза [7]:

$$
\rho=\mu=\frac{\sqrt{B} C^{\prime}}{2 \sqrt{2} C}, \quad \gamma=\varepsilon=-\frac{B^{\prime}}{4 \sqrt{2 B}}, \quad \alpha=-\beta=\frac{\operatorname{ctg} \theta}{2 \sqrt{2 C}} .
$$


С использованием этой формулы и с помощью преобразований

$$
\begin{aligned}
F_{1} & =C^{-1 / 2} R_{-1 / 2}(r) e^{-i E t} Y_{-1 / 2}(\theta, \varphi), \\
F_{2} & =R_{+1 / 2}(r) e^{-i E t} Y_{+1 / 2}(\theta, \varphi), \\
G_{1} & =R_{+1 / 2}(r) e^{-i E t} Y_{-1 / 2}(\theta, \varphi), \\
G_{2} & =C^{-1 / 2} R_{-1 / 2}(r) e^{-i E t} Y_{+1 / 2}(\theta, \varphi)
\end{aligned}
$$

получим, что уравнения для поля Дирака (3) могут быть теперь приведены к виду

$$
\begin{aligned}
& C B^{2} \frac{d^{2} R_{-1 / 2}}{d r^{2}}+\frac{\left(C B^{2}\right)^{\prime}}{2} \frac{d R_{-1 / 2}}{d r}+\left[C E^{2}+\frac{i E\left(C^{\prime} B-B^{\prime} C\right)}{2}+\right. \\
& \left.+\frac{C^{\prime} B^{\prime}+2 C B^{\prime \prime}}{8} B-\lambda^{2} B\right] R_{-1 / 2}=0, \\
& C B^{2} \frac{d^{2} R_{+1 / 2}}{d r^{2}}+\frac{\left(C B^{2}\right)^{\prime}}{2} \frac{d R_{+1 / 2}}{d r}+\left[C E^{2}+\frac{i E\left(C^{\prime} B-B^{\prime} C\right)}{2}+\right. \\
& \left.\quad+\frac{4 B C^{\prime \prime}+5 C^{\prime} B^{\prime}+2 C B^{\prime \prime}}{8} B-\lambda^{2} B\right] R_{+1 / 2}=0, \\
& \left(\frac{1}{\sin \theta} \frac{\partial}{\partial \theta}\left(\sin \theta \frac{\partial}{\partial \theta}\right)+\frac{1}{b \sin ^{2} \theta} \frac{\partial^{2}}{\partial \varphi^{2}} \pm \frac{i \cos \theta}{\sqrt{b} \sin ^{2} \theta} \frac{\partial}{\partial \varphi}+\frac{\operatorname{ctg}^{2} \theta}{4}-\frac{1}{2}+\lambda^{2}\right) Y_{ \pm 1 / 2}=0 .
\end{aligned}
$$

Уравнение $(10)$ показывает, что $Y_{ \pm 1 / 2}(\theta, \varphi)$ представляют собой сферические гармоники со спиновыми весами, а константу разделения переменных следует выбрать равной $\lambda^{2}=(l+1 / 2)^{2}[8]$. Здесь $l, m$ - целые числа, удовлетворяющие неравенствам $l \geqslant s$ и $-l \leqslant m \leqslant l$.

\section{3. КВАНТОВАЯ ЭНТРОПИЯ}

Применяя приближение Вентцеля-Крамерса-Бриллюэна с $R_{ \pm}(r)=e^{i \phi_{ \pm}(r)}$, получим, что уравнения (8) и (9) определяют радиальное волновое число $k(r, l, E) \equiv$ $\partial \phi / \partial r:$

$$
\begin{aligned}
& k_{-1 / 2}=\frac{1}{B \sqrt{C}}\left[C E^{2}+\frac{C^{\prime} B^{\prime}+2 C B^{\prime \prime}}{8} B-B\left(l+\frac{1}{2}\right)^{2}\right]^{1 / 2}, \\
& k_{+1 / 2}=\frac{1}{B \sqrt{C}}\left[C E^{2}+\frac{4 B C^{\prime \prime}+5 C^{\prime} B^{\prime}+2 C B^{\prime \prime}}{8} B-B\left(l+\frac{1}{2}\right)^{2}\right]^{1 / 2} .
\end{aligned}
$$

Условия жесткой стенки имеют вид $R_{-1 / 2}=R_{+1 / 2}=0$ при $r=r_{\mathrm{H}}+\varepsilon$ и при $r=r_{\mathrm{H}}+L$, где $0<\varepsilon \ll r_{\mathrm{H}}, L \gg r_{\mathrm{H}}$, а $r_{\mathrm{H}}=2 M$ - горизонт событий. Тогда число мод с энергиями, меньшими $E$, равно

$$
\begin{aligned}
g(E)= & \int(2 l+1) d l \frac{1}{\pi} \int_{r_{\mathrm{H}}+\varepsilon}^{r_{\mathrm{H}}+L} 2\left[k_{-1 / 2}(r, l, E)+k_{+1 / 2}(r, l, E)\right] d r= \\
= & \frac{4}{3 \pi} \int_{r_{\mathrm{H}+\varepsilon}}^{r_{\mathrm{H}}+L} \frac{C d r}{B^{2}}\left\{\left[E^{2}+\frac{B}{C}\left(\frac{\left.C^{\prime} B^{\prime}+2 C B^{\prime \prime}\right)}{8}-1\right)\right]^{3 / 2}+\right. \\
& \left.+\left[E^{2}+\frac{B}{C}\left(\frac{4 B C^{\prime \prime}+5 C^{\prime} B^{\prime}+2 C B^{\prime \prime}}{8}-1\right)\right]^{3 / 2}\right\} .
\end{aligned}
$$


Свободная энергия термического ансамбля спинорных дираковских частиц с обратной температурой $\beta$ имеет вид

$$
-\beta F=\sum_{E} \ln \left(1+e^{-\beta E}\right) .
$$

Применяя уравнение (13) для нахождения плотности состояний и опуская стандартный вклад от вакуума, окружающего систему, получим главный вклад в свободную энергию:

$$
\begin{aligned}
F=- & \frac{1}{\beta} \int_{0}^{\infty} d E \frac{d g(E)}{d E} \ln \left(1+e^{-\beta E}\right)=-\int_{0}^{\infty} \frac{e^{-\beta E}}{1+e^{-\beta E}} g(E) d E= \\
=- & \frac{7 \pi^{3}}{45 \beta^{4}} \int_{r_{\mathrm{H}}+\varepsilon}^{r_{\mathrm{H}}+L} \frac{C}{B^{2}} d r-\frac{\pi}{6 \beta^{2}} \int_{r_{\mathrm{H}}+\varepsilon}^{r_{\mathrm{H}}+L} \frac{d r}{B}\left[\frac{2 B C^{\prime \prime}+3 C^{\prime} B^{\prime}+2 C B^{\prime \prime}}{4}-2\right]= \\
=- & \frac{7 \pi^{3} r_{\mathrm{H}}^{2} C\left(r_{\mathrm{H}}\right)}{45 \beta^{4} \varepsilon}-\frac{7 \pi^{3}\left[r_{\mathrm{H}}^{2} C^{\prime}\left(r_{\mathrm{H}}\right)+2 r_{\mathrm{H}} C\left(r_{\mathrm{H}}\right)\right]}{45 \beta^{4}} \ln \frac{L}{\varepsilon}- \\
& \quad-\frac{\pi}{6 \beta^{2}}\left(\frac{3 C^{\prime}\left(r_{\mathrm{H}}\right)}{4}-\frac{C\left(r_{\mathrm{H}}\right)}{r_{\mathrm{H}}}-2 r_{\mathrm{H}}\right) \ln \frac{L}{\varepsilon} .
\end{aligned}
$$

Применяя формулу $S=\beta^{2}(\partial F / \partial \beta)$, получим квантовую энтропию:

$$
\begin{gathered}
S=\frac{28 \pi^{3} r_{\mathrm{H}}^{2} C\left(r_{\mathrm{H}}\right)}{45 \beta^{3} \varepsilon}+\frac{28 \pi^{3}\left[r_{\mathrm{H}}^{2} C^{\prime}\left(r_{\mathrm{H}}\right)+2 r_{\mathrm{H}} C\left(r_{\mathrm{H}}\right)\right]}{45 \beta^{3}} \ln \frac{L}{\varepsilon}+ \\
+\frac{\pi}{3 \beta}\left(\frac{3 C^{\prime}\left(r_{\mathrm{H}}\right)}{4}-\frac{C\left(r_{\mathrm{H}}\right)}{r_{\mathrm{H}}}-2 r_{\mathrm{H}}\right) \ln \frac{L}{\varepsilon} .
\end{gathered}
$$

Выберем обратную температуру $\beta$, отвечающую температуре Хокинга на горизонте черной дыры, т.е. $\beta=4 \pi r_{\mathrm{H}}=8 \pi M$, и положим $l_{p}^{2}=2 \epsilon^{2} / 15$ и $\Lambda^{2}=L \epsilon^{2} / \varepsilon$, где $l_{p}=\sqrt{\varepsilon \beta / \pi}$ есть собственное расстояние между жесткой стенкой и горизонтом, а $\epsilon$ и $\Lambda$ представляют собой параметры соответственно ультрафиолетового и инфракрасного обрезаний [9]. Статистически-механическая энтропия (16) при этом имеет вид

$$
S=\frac{7 A_{h}}{96 \sqrt{b} \pi \epsilon^{2}}+\frac{7}{360}\left[\frac{C^{\prime}\left(r_{\mathrm{H}}\right)}{r_{\mathrm{H}}}+\frac{2 C\left(r_{\mathrm{H}}\right)}{r_{\mathrm{H}}^{2}}\right] \ln \frac{\Lambda}{\epsilon}+\frac{1}{6}\left(\frac{3 C^{\prime}\left(r_{\mathrm{H}}\right)}{4 r_{\mathrm{H}}}-\frac{C\left(r_{\mathrm{H}}\right)}{r_{\mathrm{H}}^{2}}-2\right) \ln \frac{\Lambda}{\epsilon},
$$

где $A_{h}=4 \pi \sqrt{b} C\left(r_{\mathrm{H}}\right)$ - площадь поверхности горизонта событий.

Полагая $C=\left(1-8 \pi \eta^{2}\right) r^{2}$ и $b=1$ в выражении (17), получим энтропию поля Дирака в черной дыре с глобальным монополем:

$$
S_{\text {monopole }}=\frac{7 A_{h}^{\text {monopole }}}{96 \pi \epsilon^{2}}+\frac{7\left(1-8 \pi \eta^{2}\right)}{90} \ln \frac{\Lambda}{\epsilon}-\frac{1}{12}\left(3+8 \pi \eta^{2}\right) \ln \frac{\Lambda}{\epsilon},
$$

где $A_{h}^{\text {monopole }}=4 \pi\left(1-8 \pi \eta^{2}\right) r_{\mathrm{H}}^{2}$. Если же в выражении (17) выбрать $C=r^{2}$ и $b=1-\mu^{2}$, то получим энтропию поля Дирака в черной дыре с космической струной:

$$
S_{\text {string }}=\frac{7 A_{h}^{\text {string }}}{96 \sqrt{1-\mu^{2}} \pi \epsilon^{2}}+\frac{7}{90} \ln \frac{\Lambda}{\epsilon}-\frac{1}{4} \ln \frac{\Lambda}{\epsilon},
$$

где $A_{h}^{\text {string }}=4 \pi \sqrt{1-\mu^{2}} r_{\mathrm{H}}^{2}$. 


\section{4. ЗАКЛЮЧЕНИЕ}

Нами была исследована квантовая энтропия поля Дирака в черной дыре с глобальным монополем или с космической струной в рамках модели жесткой стенки. Уравнения (18) и (19) показывают, что, если опустить вклад от окружающей вакуумной системы, квантовая энтропия поля Дирака содержит две составляющих. Первая представляет собой собственный вклад от горизонта, и она расходится квадратично на горизонте событий. Вторая составляющая содержит два логарифмическирасходящихся члена, и эта составляющая не пропорциональна площади горизонта событий. Первые два члена в формулах (18) и (19) имеют ту же структуру, что и в случае скалярного поля; различными оказываются только коэффициенты. Однако в выражениях (18) и (19) присутствует и третий член, который задает вклад спинов дираковских частиц в энтропию, и этим членом нельзя пренебрегать как несущественной аддитивной константой. Таким образом, полное выражение не представляется в виде, аналогичном случаю энтропии скалярного поля. Этот результат сильно отличается от выводов работ [5], [6]. Отметим также, что полный логарифмический член всегда уменьшает энтропию черных дыр. Если подставить $\eta=0$ в формулу (18) или $\mu=0$ в формулу (19), то мы получим результат для черной дыры Шварцшильда.

\section{Список литературы}

[1] J.D. Bekenstein, Phys. Rev. D, 7 (1973), 2333; S. W. Hawking, Commun. Math. Phys., 43 (1975), 199.

[2] G. 't Hooft, Nucl. Phys. B, 256 (1985), 727.

[3] S. N. Solodukhin, Phys. Rev. D, 51 (1995), 609; M.H. Lee, J.K. Kim, Phys. Rev. D, 54 (1996), 3904; J. Ho, W. T. Kim, Y.J. Park, H. Shin, Class. Q. Grav., 14 (1997), 2617; R. G. Cai, L. B. Zhu, Phys. Lett. A, 219 (1996), 191; W. B. Liu, Z. Zhao, Chin. Phys. Lett., 18 (2001), 310; L.C. Zhang, Y.Q. Wu, R. Zhao, Chin. Phys., 13 (2004), 974; J. L. Jing, M. L. Yan, Chin. Phys., 9 (2000), 389; T.R. Ding, Y.Q. Wu, L.C. Zhang, Chin. Phys., 13 (2004), 268.

[4] Z.H. Li, Phys. Rev. D, 62 (2000), 024001; Mod. Phys. Lett. A, 17 (2002), 887; G. Q. Li, Acta. Phys. Sin., 52 (2003), 1346; 55 (2006), 995; Chin. Phys., 14 (2005), 468; L. Q. Mi, Acta. Phys. Sin., 53 (2004), 2065; J.L. Jing, M. L. Yan, Phys. Rev. D, 64 (2001), 064015; 63 (2001), 084028.

[5] J. L. Jing, Chin. Phys. Lett., 14 (1997), 495.

[6] M. W. Lu, Chin. J. Phys., 43 (2005), 909.

[7] E. Newman, R. Penrose, J. Math. Phys., 3 (1962), 566.

[8] Z. H. Li, L. Q. Mi, Acta. Phys. Sin., 48 (1999), 575.

[9] S. N. Solodukhin, Phys. Rev. D, 51 (1995), 618. 